\title{
DE LA DOBLE VERSIÓN AL POEMA FANTASMA: LA CREATIVIDAD DE LA IMPRENTA*
}

\author{
Maria Mercè LóPez Casas \\ Universidade de Santiago de Compostela
}

El nacimiento de la imprenta supuso un cambio sustancial en la cultura del hombre de fines del siglo XV y sobre todo del XVI. Se abrió una nueva dimensión que llevó a una Europa diferente, de manera semejante a lo que ha supuesto para nosotros, hombres y mujeres del siglo XXI, el nacimiento de internet, que nos augura una nueva era para la humanidad, abriendo perspectivas, formas distintas de adquisición de conocimientos y nuevos modos de relación entre personas, sin siquiera soñar las novedades e ingenios del futuro que nos aguarda. Del mismo modo que la concepción del libro -incluso de la literatura de creación- ha mutado para nosotros, cambió la forma y el sentido del libro para el hombre del siglo XVI con el impreso.

En los primeros tiempos de la imprenta la producción religiosa fue predominante: ediciones de la Biblia, misales, breviarios, salterios, así como otro tipo de impresos derivados de las autoridades eclesiásticas como las actas de capítulos provinciales, constituciones sinodales, catecismos o bulas de indulgencia. Desde el período incunable se editaron también tratados de derecho y recopilaciones legislativas, normas dictadas por los reyes y emanadas de otros órganos gubernamentales. No debemos olvidar los miles de ejemplares de manuales de lengua que fueron estampados, fundamentalmente el Ars minor de Donato en el que se basó durante toda la Edad Media la enseñanza y el uso del latín ${ }^{1}$. A esos manuales procedentes de siglos anteriores se añadieron pronto otros ya pensados para la imprenta, como por ejemplo las Introductiones Latinae de Antonio de Nebrija (veinte ediciones) o los primeros vocabularios y diccionarios como el Vocabolista italiano-tedesco (Venecia, Adam

${ }^{*}$ Este trabajo se enmarca en el proyecto La variante en la imprenta: hacia un canon de transmisión del cancionero y del romancero medievales, financiado por el Ministerio de Economía y Competitividad (FFI2011-25266), coordinado por Josep Lluís Martos como investigador principal.

${ }^{1}$ También se estampó con frecuencia el Doctrinale de Alexander de Villa Dei. 
von Rottweil, 1477) o el Vocabolari molt profitos per apendre Lo Catalan Allamany y Lo Allamany Catalan (Perpinyà, Johan Rosembach, 1502) 2 .

Pero hubo otro tipo de obras que interesaron al público lector europeo, público que, por otra parte, no puede decirse que constituyera un bloque homogéneo ni social ni cultural ni topogeográfico. El repertorio estaría formado por las obras historiográficas, las de la literatura clásica y humanística, las espirituales y de normas de conducta moral, prosa de ficción y poesía narrativa y lírica. En ese conjunto variopinto de obras, en los talleres tipográficos europeos ocuparon un lugar destacado los textos medievales, incluso hasta las últimas décadas del siglo Xvi. Nos hallamos ante una confluencia de caminos: la creación (el texto medieval) y su difusión (el libro impreso). Lo que suscita varias cuestiones para la reflexión: 1) ¿Qué tipo de textos literarios medievales se siguieron leyendo impresos? y 2) ¿Cómo y de qué manera pasaron a la imprenta? ${ }^{3}$ Dicho de otro modo, ¿cómo se transmitió la literatura medieval a los libros impresos? Este planteamiento implica además abordar todo tipo de traducciones, reelaboraciones, refundiciones y adaptaciones (incluyendo la actualización lingüística, pongamos como ejemplo la impresión de las obras de Ramon Llull, autor del siglo XIII en la Valencia del siglo XVI) ${ }^{4}$. El tema es complejo porque a su vez nos obliga a revisar otro tipo de materias: $¿$ en qué medida la producción impresa afectó a los géneros literarios y a sus límites? Lo cierto es que la línea entre historia y ficción fue desdibujándose; estoy pensando por ejemplo en la proximidad de personajes épicos a héroes caballerescos ${ }^{5}$, como sucede con las ediciones cidianas del siglo XVI, o en la

${ }^{2}$ Hay facsímile del Vocabulari confeccionado sobre el único ejemplar conocido, el que perteneció al erudito Marià Aguiló, custodiado en la Biblioteca de Cataluña. La reproducción facsimilar va acompañada de otro volumen con varios estudios, Postil·les / Randbemerkungen, realizados por Dolors Lamarca, Pere Barnils, Tilbert Dídac Stegmann y Vinyet Panyella, Barcelona, Biblioteca de Catalunya - Institut Ramon Llull, 2002.

${ }^{3}$ Aborda el tema José Simón Díaz con un estudio pionero titulado «La literatura medieval castellana y sus ediciones españolas de 1501 a 1560», en El libro antiguo español. Actas del primer Coloquio Internacional (Madrid, 18 al 20 de diciembre de 1986), ed. M. ${ }^{a}$ Luisa López-Vidriero y Pedro M. Cátedra, Salamanca - Madrid, Ediciones de la Universidad de Salamanca - Biblioteca Nacional de Madrid - Sociedad Española de Historia del Libro, 1988, pp. 371-396.

${ }^{4}$ Blanquerna: qui tracta de sinch estaments de persones de Matrimoni de Religió de Prelatura I hordenat per... Ramon LLull; traduit y corregit ara novament dels primers originals... en llengua Valenciana [per Joan Bonlabij]; ab lo Libre de oracions y contemplacions del enteniment en deu per lo matex doctor. Estampado en Valencia, en casa de Joan Jofre, 1521. Fue su editor Gregori Genovart, canónigo de la Seo de Mallorca según consta en el colofón. Véase sobre este impreso y su actualización lingüística (adaptada a la lengua de Valencia) Beatrice Schmid, Les «traduccions valencianes» del Blanquerna (València 1521) i de la Scala Dei (Barcelona 1523). Estudi lingüistic, Barcelona, Curial-PAM, 1988. Para una valoración más general del período en relación al catalán, véase Antoni Ferrando, «El paper dels primers editors (1473-1523) en la fixació del catalá modern», Caplletra, 27 (1999), pp. 109-136.

${ }^{5}$ Los textos históricos de nueva creación o refundidos salían de las mismas prensas y con características semejantes a los libros de caballerías en virtud del interés de impresores y libreros. 
novelización de episodios de la conquista de Granada ${ }^{6}$. Asimismo surgieron géneros ligados directamente a la imprenta, lo que se ha venido en llamar «géneros editoriales» ${ }^{7}$, algunos con raíces ancladas en la Edad Media, y no me refiero solo a la prosa de ficción (ciclos narrativos al estilo de los libros de caballerías, narrativa caballeresca breve, literatura celestinesca, o relaciones de sucesos) sino también al romancero, estudiado en dicho sentido por Alejandro Higashi, quien reclama esta etiqueta para el romancero impreso de los siglos XVI y XVII, gracias a la labor de Martín Nucio, «impresor responsable de convertir las diferentes compilaciones previas de romances en un género editorial exitoso» ${ }^{8}$. En cuanto a la poesía de cancionero, creada en ámbitos cortesanos, gracias a la imprenta se difundió a capas más amplias de la población. En palabras de Manuel Moreno «la imprenta es quien hace moda de la poesía de cancionero» ${ }^{9}$, y para ello fue fundamental la publicación en 1511 del Cancionero general de Hernando del Castillo, la magna colección de más de mil poemas, que tuvo notable éxito entre el público urbano, con diversas reediciones: desde su aparición en 1511 hasta 1573 se hicieron nueve ediciones, en Valencia, Toledo, Sevilla y Amberes.

Y a todos estos cambios que vive la literatura medieval en su transmisión impresa -y en su recepción- habría que incorporar un nuevo concepto, con repercusiones textuales, el de la «materialidad», entendido como el conjunto

${ }^{6}$ Carmen Parrilla, a partir del interés de Cervantes -reflejado en el Quijote- por la producción impresa de su época, analiza los modelos histórico-literarios plasmados no solo en las novelas de su tiempo sino también en la literatura histórica de nueva creación y en la recuperación de crónicas y biografías (El Cid o Bernardo del Carpio entre otros), lo que revela un marco mucho más complejo, un juego de relaciones entre las diversas obras que difundían los impresores del Siglo de Oro. Véase Carmen Parrilla, «Libros de caballerías en El Quijote. Lectura y lectores ¿el texto espejo?», Boletín de la Biblioteca de Menéndez Pelayo, 81 (2005), pp. 329-362.

${ }^{7}$ Acuñó la expresión terminológica Víctor Infantes en su artículo «El género editorial de la narrativa caballeresca breve», Voz y Letra, 7:2 (1996), pp. 127-132. Véase también íd., «Los géneros editoriales: entre el texto y el libro», en La cultura del libro en la Edad Moderna. Andalucía y América, ed. Manuel Peña Díaz, Pedro Ruiz Pérez y Julián Solana Pujalte, Córdoba, Universidad de Córdoba, 2001, pp. 37-46.

${ }^{8}$ Alejandro Higashi, «El género editorial y el Romancero», Lemir, 17 (2013), pp. 37-64, donde realiza un minucioso análisis del proceso desde el romancero pre-editorial hasta el Cancionero de romances de Martín Nucio. Véase también íd., «Función de la microvariante: del pliego suelto al Cancionero de romances», en La poesía en la imprenta antigua, ed. Josep Lluís Martos, Alacant, Universitat, 2013, pp. 309-328. Véase al respecto también el trabajo de Paloma Díaz-Mas, «El romancero, entre la tradición oral y la imprenta popular», en destiempos.com, 15:1 (2008), pp. 115-129, y el de Vicenç Beltran, «El romancero: de la oralidad a la imprenta», en La poesía en la imprenta, ob. cit., pp. 251-267. Sobre el Cancionero de romances y Martín Nucio en relación con la imprenta, véase Josep Lluís Martos, «E1 público de Martín Nucio: del Cancionero de romances al Cancionero general de 1557», en Convivio. Cancioneros peninsulares, ed. Vicenç Beltran y Juan Paredes, Granada, Universidad, 2010, pp. 111-124, e íd., «La autocensura en los cancioneros: una justificación impresa en 57CG y otra manuscrita en CT1», en Cultura Neolatina, 70:3-4 (2010), pp. 155-180.

${ }^{9}$ Manuel Moreno, «El canon de Mena en la imprenta», en La poesía en la imprenta, ob. cit., pp. 121136; la cita en las pp. 129-130. 
de factores que en el proceso de la impresión condicionan la forma final del texto, y que permiten explicar el origen de no pocas alteraciones, es decir, de la variación o de determinado tipo de variantes que solo hallamos en las ediciones por su vinculación al proceso impresor ${ }^{10}$.

Me voy a ocupar en este trabajo de un poeta del siglo XV que tuvo gran prestigio en su época, pues fue admirado por sus coetáneos, catalanes y castellanos. El Marqués de Santillana, en el Prohemio e carta, le dedicó las siguientes palabras: «grand trobador e omne de asaz eleuado spiritu» ${ }^{11}$. De igual manera fue ensalzado, venerado e imitado por los autores hispánicos de los siglos XVI y XVII ${ }^{12}$. Me estoy refiriendo a Ausiàs March ${ }^{13}$. Joan Boscà o Boscán, uno de sus más firmes admiradores, cuya autoridad y preeminencia sobre sus

\footnotetext{
${ }^{10}$ Véase el artículo de Jaime Moll, «Problemas bibliográficos del libro del Siglo de Oro», Boletín de la Real Academia Española, 59: 216 (enero-abril 1979), pp. 49-107. La materialidad en la transmisión del romance ha sido el caso mejor estudiado y que más frutos ha dado, desde las aportaciones de Giuseppe Di Stefano («Edición crítica del romancero antiguo: algunas consideraciones», en Actas del Congreso Cancionero-Romancero,UCLA, 1984, ed. Enrique Rodríguez Cepeda, con la colaboración y bibliografía crítica de Samuel G. Armistead, Madrid, Porrúa Turanzas, 1990, pp. 29-46; íd., «El pliego suelto, del lenguaje a la página», en Imprenta y Crítica Textual en el Siglo de Oro, coord. Francisco Rico, ed. Pablo Andrés Escapa y Sonia Garza Merino, Valladolid, Universidad, Centro para la Edición de los Clásicos Españoles, 2000, pp. 171-185; íd., «El impresor-editor y los romances», en La literatura popular impresa en España y en la América colonial. Formas y temas, géneros, funciones, difusión, historia e teoria, Salamanca, SEMYR, 2006, pp. 415-424). Hasta las de Mario Garvin (Scripta Manent. Hacia una edición crítica del romancero impreso (Siglo XVI), Madrid-Frankfurt, Iberoamericana, 2007). Aunque Garvin va un paso más allá afirmando que «centrar el estudio de la variación únicamente en la variante textual supone pasar por alto precisamente el factor que la condiciona. Habrá que concluir entonces que al igual que la performance es parte esencial y constitutiva del romance oral, el romancero impreso no está únicamente en el texto, sino que su ontología reside en la interacción de los dos códigos semióticos, el lingüístico y el material» (Mario Garvin, «La lógica del pliego suelto. Algunos apuntes sobre la materialidad en la transmisión poética», en La poesía en la imprenta, ob. cit., pp. 295-308, p. 299.

${ }^{11}$ Marqués de Santillana, El Prohemio e carta del Marqués de Santillana y la teoría literaria del s. XV, ed. de Ángel Gómez Moreno, Barcelona, PPU, 1990, p. 60. Véase también Lluís Cabré, «Notas sobre la memoria de Santillana y los poetas de la Corona de Aragón», en "Cancionero» Studies in Honour of Ian Macpherson, ed. Alan Deyermond, London, Department of Hispanic Studies - Queen Mary and Westfield College, 1998, pp. 25-38, y Rafael Mérida Jiménez, «Ausiàs March, matèria de poètica i debat (de Santillana a Lope de Vega)», en Canelobre. Estudis sobre Ausiàs March, 39-40 (1998-1999), pp. 211-216.

${ }^{12}$ En lo que se refiere al ámbito catalán, véase Eulàlia Duran, «Defensa de la pròpia tradició davant d'Itàlia al segle XvI» en Miscel·lània Joan Fuster III, Barcelona, PAM, 1991, pp. 241-265, y de la misma autora, «La valoració renaixentista d'Ausiàs March», en Homenatge a Arthur Terry I. Estudis de Llengua i Literatura Catalanes, 35 (1997), pp. 241-265. Y también Joan Fuster, «Lectures d'Ausiàs March en la València del segle XvI», en Estudi General. Homenatge a Joan Fuster, 4 (1984), pp. 31-55.

${ }^{13}$ En catalán se ha llegado a acuñar el término «ausiasmarquista», referido a los poetas que siguen o imitan a Ausiàs March, "poetes ausiasmarquistes», como fueron Pere Torroella, Hug de Rocabertí, Romeu Llull, Pedro de Urrea, Joan Roís de Corella, Joan Ferrandis d'Herèdia, Francesc Ferrer y Hugo de Urriés entre otros. Véase Lluís Cabré, «From Ausiàs March to Petrarch: Torroella, Urrea, and other Ausiamarchides», en The Medieval Mind. Hispanic Studies in Honour of Alan Deyermond, ed. Ian Macpherson y Ralph Penny, London, Tamesis, 1997, pp. 57-74; y Francisco J. Rodríguez Risquete, «El mestratge de Pere Torroella», en Actes del Tretzè Col-loqui Internacional de Llengua i Literatura Catalanes (Girona 2003), ed. Sadurní Martí et alii, Barcelona, PAM, 2007, III, pp. 337-362.
} 
compañeros poetas fijó la posición de magisterio de Ausiàs March, le llamó «el grande catalán de amor maestro» ${ }^{14}$, además de encumbrarle en la epístola a la Duquesa de Soma como «el más excelente autor catalán dentre los provenzales» ${ }^{15}$. Si Juan de Mena fue el poeta famoso y famosísimo en sus ediciones quinientistas, la primera edición de la poesía de Ausiàs March, estampada en Valencia en 1539, tiene por título Las obras del famosissimo philosofo y poeta mossen Osias Marco ${ }^{16}$. Porque la imprenta tuvo mucho que ver con el éxito y la reivindicación de Ausiàs March como poeta en el siglo XVI. Y si los impresos de las obras de March a priori podrían parecer fuentes de carácter secundario, su estudio bibliográfico y textual revela que presentan novedades con respecto a los testimonios más antiguos, los manuscritos del siglo xV.

En este artículo voy a centrarme en los poemas «nuevos» que aportan

${ }^{14}$ El texto entresacado de la siguiente estrofa (Boscán, Obras, ed. de Carlos Clavería, Barcelona, PPU, 1991, p. 560):

$$
\begin{aligned}
& \text { Y al grande catalán, d amor maestro } \\
& \text { Aosias March, que en su verso pudo tanto. } \\
& \text { que enrriqueció su pluma el nombre nuestro } \\
& \text { con su fuerte y sabroso y dulce llanto. }
\end{aligned}
$$

${ }^{15}$ Tomo la referencia de Vicent Josep Escartí, La primera edició valenciana de l'obra d'Ausiàs March 1539, València, Bancaixa - Universitat de València - Generalitat Valenciana - Biblioteca Nacional, 1997, p. 48. En cuanto al predicamento de Ausiàs March, así como el papel de Boscán como transmisor de su poesía a los poetas del primer Renacimiento castellano, me remito solamente a los estudios de conjunto clásicos, Martí de Riquer, «La influencia de Ausiàs March en la lírica castellana de la Edad de Oro», Revista Nacional de Educación, 8 (1941), pp. 49-74; Juan Manuel Rozas, «Petrarca y Ausiàs March en los sonetos-prólogo amorosos del Siglo de Oro», en Homenajes. Estudios de Filología Española, 1964, I, pp. 57-75; Rafael Ferreres, «La influencia de Ausiàs March en algunos poetas del Siglo de Oro», en Estudios sobre literatura y arte dedicados al profesor Emilio Orozco Díaz, ed. Antonio Gallego Morell, Andrés Soria y Nicolás Marín, Granada, Universidad, 1979, I, pp. 469-483; Kathleen McNerney, «Ausias March and Juan Boscan», en Actes del Primer Col-loqui d'Estudis Catalans a Nord-Amèrica, Barcelona, PAM, 1979, pp. 195-209, y de la misma autora, The Influence of Ausiàs March on Early Golden Age Castilian Poetry, Amsterdam, Rodopi, 1982. Véase también Marinela Garcia Sempere, «La relació de l'obra poètica de Joan Boscà amb la d'Ausiàs March», en Estudis de Llengua i Literatura Catalanes, XXXIII. Miscel·lània Germà Colon, 6 (1996), pp. 89-108.

${ }^{16} \mathrm{La}$ edición llevada a cabo por Baltasar de Romaní es a la vez la primera traducción castellana, ya que en cada poema a cada estrofa catalana le sigue su correspondiente versión castellana. Reúne solamente 46 poemas. El volumen fue estampado en Valencia por Juan Navarro el 10 de marzo de 1539. Hay facsímil de la edición valenciana de Romaní acompañado de estudio y edición por Escartí, La primera edició, ob. cit., Sobre este impreso, véase también Maria Mercè López Casas, «La recepció d'Ausiàs March al segle xvi: l'edició de Romaní (1539)», Caplletra, 34 (2003), pp. 79-110, y Albert Lloret, Printing Ausiàs March. Material Culture and Renaissance Poetics, Madrid, Centro para la Edición de los Clásicos Españoles, 2013, pp. 17-99. En cuanto al interés de Ausiàs March como filósofo, surge en el marco de la polémica renacentista sobre las artes, propiciada por el mejor conocimiento de la Poética de Aristóteles, según ha señalado Duran, «Defensa de la pròpia tradició», art. cit., pp. 95-96. La valoración aristotélica de la filosofía por encima de la poesía se basaba en la capacidad de la filosofía para tratar temas universales. La poesía de Ausiàs March se convierte en texto de filosofía moral, las «moralidades de Osias Marco» que cita Baltasar de Romaní en la epístola dirigida al Duque de Calabria; es la manera humanística de prestigiar la poesía. 
las ediciones, más concretamente en aquellos textos que, en cierta medida, podrían haber sido configurados por el editor, de ahí la expresión de «poema fantasma» que reza en el título; poemas que quizás no escribió Ausiàs March en la forma en que nos han llegado. Y para hacerlo me basaré por una parte en criterios textuales, y por otra $-\mathrm{y}$ complementaria- en los usos y costumbres de los talleres tipográficos, esto es, teniendo en cuenta también la manera de trabajar de los editores del siglo XVI. Concretamente estos poemas son el 102 b y la tercera versión del poema 103, que aparecen por vez primera en la edición de Juan de Resa - conocida con la sigla $d$ - la estampada en Valladolid por Sebastián Martínez en $1555^{17}$; textos después recogidos en la edición de Barcelona de Claudi Bornat, 1560 (impreso $e)^{18}$.

Los cancioneros quinientistas que nos han transmitido los poemas de March, tanto manuscritos como impresos, excepto la primera edición de 1539 de Baltasar de Romaní, responden a la idea de recopilar toda la obra del poeta, con clara intención de reconstruir -restaurar- el «Cancionero de Ausiàs March», restauración que ha de ser entendida desde una triple perspectiva: a) recopilar todos y cada uno y solo los poemas de March y completar la colección dispersa de su poesía ${ }^{19}$ (lo que incluiría la reordenación de los materiales); b) colacionar distintas versiones de poemas y versos, y c) seleccionar fragmentos o lecciones consideradas originales ${ }^{20}$. El prólogo del manuscrito de Lluís Carròs de Vilaragut -conocido con la referencia $E$ - es revelador en este sentido ${ }^{21}$ :

${ }^{17}$ LAS OBRAS DEL POETA MO| sen Ausias March, corregidas de los errores que | tenian. Sale con ellas el vocabulario de los vo |cablos en ellas contenidos. Dirigidas al Illus | trissimo señor Gonçalo. Fernandez de Cor |doua, Duque de Sesa, y de Terranoua, Con |de de Cabra... Valladolid, en casa de Sebastián Martínez, 20 de febrero de 1555. Hay reproducción facsimilar de esta edición en Els poemes d'Ausiàs March. Edició Valladolid, 1555, ed. de José Vicente Gómez y Voro López i Verdejo, València, Real Acadèmia de Cultura Valenciana, 2002.

${ }^{18}$ Sigo la nomenclatura de Amadeu Pagès, Les obres d'Auzias March, Barcelona, Institut d'Estudis Catalans, 1912-14, 2 vols., tanto para citar los testimonios como para la numeración de los poemas. Numeración que encontramos también en la edición de Robert Archer de Ausiàs March. Obra completa, Barcelona, Barcanova, 1997, 2 vols.

${ }^{19}$ Sobre todo si tenemos en cuenta que parte de la obra de Ausiàs March se difundió a partir de cuadernos sueltos, como ha demostrado el estudio de Vicenç Beltran, Poesia, escriptura i societat: els camins de March, Castelló - Barcelona, Fundació Germà Colón Domènech - PAM, 2006, pp. 74-87, en lo que concierne a los poemas 101-128.

${ }^{20}$ Véase Josep Lluís Martos, «La restauración de las obras de Ausiàs March: los cancioneros impresos del siglo XVI», en I Canzonieri di Lucrezia / Los Cancioneros de Lucrecia, ed. Andrea Baldissera y Giuseppe Mazzocchi, Ferrara, Unipress, pp. 409-425, e íd., «La copia completa y la restauración parcial: los cancioneros impresos de Ausiàs March en los manuscritos», en Del impreso al manuscrito en los cancioneros, ed. Josep Lluís Martos, Alcalá de Henares, Centro de Estudios Cervantinos, 2011, pp. 13-45.

${ }^{21}$ El cancionero $E$ es una copia manuscrita ejecutada por Jeroni Figueres el 1 de mayo de 1546. Lluís Carròs de Vilaragut ordenó confeccionarlo a petición de su esposa Àngela Borja i Carròs de Vilaragut, muy aficionada a las obras de Ausiàs March, según nos dice en el prólogo. Para la descripción e historia 
hauent legit | vist y reconegut molts libres antichs escrits de ma $\mid$ per los contemporals amb lo dit auctor verifficant $\mid$ y comprobant los vns ab los altres y ab les dos $\mid$ jmpressions fetes en Barcelona per manament del |jllustre Admirant de Napols don ferrando de Cardona la una a xxij de dehembre Any M.D.XXXXIII y | laltra en lo mateix dia y mes, Any M.D.XXXXV| vistes les errors e inaduertencies dels impressors les | quals corrompen la escriptura y sentencies de les dites obres, feta diligencia per molts anys en tot lo que es estat | a mi posible axi per complir los manaments | y satisfer la voluntat de la dessus dita dama y senyora $\mid$ pera que son desig fos complidament satisfeta com $\mid$ per que de tal auctor restas perpetua memoria y $\mid$ verdadera scriptio ${ }^{22}$.

\section{Lluís Carròs afirma haber acudido a manuscritos antiguos, es decir, que} fueron copiados en época del autor, haberlos cotejado unos con otros y con los dos impresos de Barcelona, que el compilador atribuye al patrocinio de Ferran Folc de Cardona ${ }^{23}$. Que una vez cotejados, advirtió los errores y olvidos de los

del manuscrito, véase Pagès, Les obres, ob. cit., I, pp. 28-31; Xavier Dilla, «El manuscrit $E$ d'Ausiàs March: ordre alfabètic o "reimaginació"?» en La Cultura Catalana tra l'Umanesimo e il Barocco. Atti del v Convegno dell'Associazione Italiana di Studi Catalani (Venezia, 24-27 marzo 1992), ed. Carlos Romero y Rossend Arqués, Padova, Programma, 1994, pp. 219-228; Vicenç Beltran, Poesia, escriptura $i$ societat, ob. cit., pp. 157-159, BITECA Manid 1203; y M. ${ }^{a}$ Mercè López Casas, «De los impresos al cancionero E de Ausiàs March», en Del impreso al manuscrito, ob. cit., pp. 173-177.

${ }^{22}$ Folio v. La transcripción es mía.

${ }^{23}$ Las ediciones, supuestamente auspiciadas por Ferran Folc de Cardona, se realizaron en el taller tipográfico de Carles Amorós en 1543 y en 1545, impresos $b$ y $c$ respectivamente. Cada vez tengo más dudas sobre si Folc de Cardona fue o no patrocinador de dichas ediciones, pese a que tanto en Valencia (queda claro en el Cancionero de Carròs de Vilaragut) como en Barcelona (por lo que dice en el prólogo su editor Claudi Bornat), había dicha creencia. De que Folc de Cardona era un admirador y recopilador de las obras de Ausiàs March no hay duda alguna, lo dice el propio Boscán en la epístola a la duquesa de Soma, la esposa de Folc de Cardona: «Mas basta para esto el testimonio del señor Almirante, que después que vio una vez sus obras las hizo luego escribir con mucha diligencia y tiene el libro dellas por tan familiar como dizen que tenía alexandre el de Homero (tomo la referencia y transcripción de Escartí, $L a$ primera edició, ob. cit., p. 48). Además dos cancioneros manuscritos con los poemas de Ausiàs March fueron de su propiedad ( $B$ y $K$, cuya copia fue encargada por él a Pere de Vilasaló, quien los ejecutó en 1541 y 1542 respectivamente), sin embargo ninguno de estos manuscritos fue tenido en cuenta para la preparación de las ediciones estampadas en el taller de Carles Amorós, al contrario se tomó como original para la imprenta el manuscrito $D$, que fue revisado y corregido en el taller sin tener en cuenta lección alguna de los manuscritos que tenía en propiedad Folc de Cardona en su casa. Por otro lado, no hay ninguna mención a Folc de Cardona en los propios impresos ni figura su escudo nobiliario, como solía hacerse en las ediciones auspiciadas por un mecenas o patrocinador, lo cual es raro siendo Cardona uno de los notables del reino. Las fuentes documentales aconsejan prudencia también (me refiero a los textos publicados por Josep M. Madurell y Jordi Rubió en Documentos para la historia de la imprenta y librería en Barcelona (1474-1553), Barcelona, Gremio de Editores, de Libreros y de Maestros Impresores, 1955). He tratado esta cuestión con detalle en «¿El cancionero $D$ de Ausiàs March, un original de imprenta?» en Actas del XIII Congreso Internacional de la Asociación Hispánica de Literatura Medieval. In memoriam Alan Deyermond, ed. José Manuel Fradejas Rueda, Deborah Dietrick, D. Martín Sanz

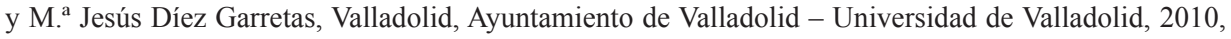
II, pp. 1181-1200 (esp. pp. 1197-1199), pero por aquel entonces yo me inclinaba a pensar que efectivamente las ediciones $b$ y $c$ podían estar relacionadas con Folc de Cardona como considera la crítica; sin 
impresores, esto es, lagunas de texto y omisión de poemas ${ }^{24}$.

La edición posterior a este manuscrito es la de 1555, estampada en Valladolid, y se incardina plenamente en el proyecto de reconstruir la totalidad del Cancionero de Ausiàs March. En sus prolegómenos figura el privilegio que obtuvo Juan de Resa para editar y vender el libro en Castilla durante 10 años, donde además se nos dice

vos con di |ligencia auiades buscado y $\mid$ copilado las obras de Au- sias March, poeta Español. |Y las auiades sacado de exemplares mas verda| deros que estauan en la impression que de las dichas | obras se auian hecho en Barçelona ${ }^{25}$.

También Jorge de Montemayor, en el prólogo de su traducción de la poesía de Ausiàs March, afirma haber visto cinco originales ${ }^{26}$. No tiene pues que extrañarnos que cada vez que empiece un nuevo proyecto editorial haya la voluntad «humanística» de volver a la pureza original del Cancionero de March, así como el propósito de buscar más obras del autor, que podían estar dispersas para incluirlas en la edición que se iba a confeccionar en el taller. Y eso es lo que hizo efectivamente Juan de Resa, ya que el impreso de Valladolid nos ofrece por primera vez el texto completo de los poemas 117 y 126 (con las «tornadas») la versión más larga del poema 68 , y una nueva «esparsa» creada,

embargo, ahora creo que por algún motivo que desconocemos no se fraguó dicho proyecto, aunque las obras de Ausiàs March llegaron a la imprenta igualmente. Sería necesario exhumar más documentación que permitiera inclinar la balanza hacia un lado u otro en este asunto.

${ }^{24}$ Lagunas como las que encontramos en el poema 116, en el que faltan la séptima y undécima estrofa, así como los dos últimos versos de la composición (vv. 151-152). En el 119 tampoco figuran ni la segunda ni la quinta estrofa ni el dístico final del poema (vv. 101-102). También han sido omitidos los poemas 12, 124, 125, 127 y 128 en los impresos de Barcelona. Para la pérdida de dichas estrofas y la omisión de los poemas, que responden a distinta casuística, véase Albert Lloret «La formazione di un canzoniere a stampa», Ecdotica, 5 (2008), pp. 103-125. Véase asimismo López Casas, «¿El cancionero $D »$, art. cit., especialmente pp. 1185-1187.

${ }^{25}$ Folio 2. La transcripción es mía.

${ }^{26}$ «Cinco originales he visto / deste Poëta, y algunos diffieren en la / letra de ciertas estanças, por donde la $\mid$ sentencia quedaua confussa en algo. Yo $\mid$ me he llegado mas al que hizo tresla- $\mid$ ladar el señor don Luis Carroz Bayle | general desta ciudad: por que segun to | dos lo affirman, el lo entendio mejor que ninguno de los de nuestros tiempos», folio 3 (la transcripción es mía).

La traducción de Jorge de Montemayor está relacionada textualmente con el texto que presenta Juan de Resa en su edición; los cinco originales a los que se refiere Montemayor fueron muy probablemente los mismos que vio Juan de Resa. He tratado esta cuestión en «Ausiàs March traducido por Jorge de Montemayor: la edición valenciana de 1560», en «Pola melhor dona de quantas fez Nostro Senhor». Homenaxe á Profesora Giulia Lanciani, coord. Mercedes Brea, Santiago de Compostela, Xunta de Galicia, Secretaría Xeral de Política Lingüística, Centro Ramón Piñeiro para a Investigación en Humanidades, 2009, pp. 291-311, y en «Los Cantos de Amor de Ausiàs March traducidos por Jorge de Montemayor», en Estudios de literatura medieval. 25 años de la AHLM, ed. Antonia Martínez Pérez y Ana Luisa Baquero Escudero, Murcia, Universidad, 2012, pp. 519-527. 
según parece, a partir del poema 102, además de incluir una versión distinta del poema 103. La edición de Juan de Resa, no obstante, siguió el texto de los impresos barceloneses para preparar su edición, acomodándose a los usos y costumbres de los talleres impresores de la época. El maestro del taller necesitaba un "original de imprenta", un manuscrito concebido para ser empleado como modelo para preparar la edición ${ }^{27}$. Cuando ya había una edición anterior a aquella que se estaba gestando, la práctica común era utilizarla como original de imprenta, y ese es el procedimiento que se siguió en Valladolid, en el taller de Sebastián Martínez, uno de los impresores más importantes de la ciudad, donde aún residía la corte. La base y punto de partida fueron los impresos de Barcelona ${ }^{28}$, no obstante, sí es cierto que usó lecciones de otros testimonios, y que reordenó algunas composiciones creando, por ejemplo, una nueva sección de carácter formal; al final de los «cantos de amor» figuran las «esparças de amor» espigadas del lugar que ocupaban en las «obres de amor» en los impresos barceloneses ${ }^{29}$. El orden de los poemas en el impreso de Valladolid es básicamente el mismo que el de las ediciones de Barcelona, pero la división de poemas en grupos es diferente. Mientras que los poemas en los impresos barceloneses sólo se agrupaban en «obres de amor», «obres de mort» y «obres morals», la edición de Valladolid presenta división en «cantos de amor» seguidos de las «esparças de amor» ${ }^{30}$, «obras morales», «canto espiritual» $\mathrm{y}$ «obras de muerte». Juan de Resa recupera en cierta forma la «cantica espiritual» de la edición valenciana de Romaní, aunque en el impreso vallisoletano esta sección solamente está formada por el poema 105.

A pesar de que, como he dicho, la edición $d-\mathrm{y} e$, la edición barcelonesa de Claudi Bornat, que es deudora del impreso vallisoletano- leen en su mayor parte como los impresos de Barcelona en los que se basan, hay algunos casos

${ }^{27}$ Véase Pablo Andrés Escapa et al., «El original de imprenta», en Imprenta y crítica textual, ob. cit., pp. 29-64. Véase también al respecto Francisco Rico, «Cómo se hacía un libro en el Siglo de Oro», El texto del "Quijote”. Preliminares a una ecdótica del Siglo de Oro, Barcelona, Destino, 2005, pp. 58-59, especialmente las notas 11 y 12.

${ }^{28}$ Ya había sido señalado por Martos: «el cancionero de Valladolid sigue dependiendo del que llevó a las prensas Carles Amorós en 1543. Resa quiso perfeccionarlo y avanzar en la restauración del cancionero de March y, aunque lo hizo a partir de las ediciones de Barcelona, también utilizó otros materiales, como él mismo advierte en el prólogo» (Martos, «La restauración de las obras», art. cit., p. 421).

${ }^{29}$ Ibídem, p. 421. Quizás influido por la disposición de algunas «esparsas» en el Cancionero $E$; véase lo que he tratado al respecto en «De los impresos al cancionero E... », p. 179.

${ }^{30}$ En los manuscritos que presentan rúbricas figura también esparça encabezando cada uno de dichos poemas que constituyen el grupo de las esparças de amor; pero como se puede observar no está el complemento nominal damor, solo hay la palabra esparça. Como en algunos casos, en el margen superior de los folios, consta la rúbrica obres damor podría pues quedar sobreentendido que se trata de esparsas de amor. 
en que las ediciones $d e, \mathrm{y}$ a veces sólo $d^{31}$, leen como los otros testimonios que no están relacionados ni derivan del manuscrito $D$ (que es antígrafo de $b c)^{32}$, lo que nos permite afirmar y concluir que efectivamente Juan de Resa consultó otras fuentes distintas de la tradición de $D$ que le llegó a través de los impresos $b c^{33}$. En los estudios sobre Ausiàs March se ha venido relacionando la edición $d$ con el cancionero $E$, ya que se consideraba que este fue compuesto por Lluís Carròs de Vilaragut como manuscrito preparatorio para la imprenta y, aunque el proyecto se frustró, indirectamente llegó a las prensas de Valladolid ${ }^{34}$. Sin duda influyó en esta idea de los investigadores que la vida de Ausiàs March que figura en los prolegómenos del impreso vallisoletano parece ser un compendio de la biografía que hay en el cancionero $E$, y puede que también influyera la ordenación alfabética de los poemas que impuso el compilador de $E$, ajena a la tradición marquiana. Parece que Juan de Resa tuvo entre sus manos el cancionero $E$ o una copia de él; se infiere por la biografía de March que hay en ambos testimonios ( $d$ resume la de $E$,

\footnotetext{
${ }^{31}$ Porque la edición $e$, estampada en Barcelona por Claudi Bornat, recupera lecciones de las ediciones barcelonesas, que Bornat vinculaba al mecenazgo de Ferran Folc de Cardona. El dedicatario de la edición $e$ no es otro que Folc de Cardona, y Bornat se erige como continuador de la labor de recuperación de las obras del poeta iniciada por el Gran Almirante: «he volgut a ymitació de la vostra senyoria continuar la instauració de les obres de Ausiàs March, ab fer-les novament estampar en ma casa lo més correcte que és estat possible reglant aquelles y ajustant-hi algunes coses que eren estades omeses [...] vostra senyoria il-lustríssima sie servit de legir-les y alegre's de veure-les correctes y aprés de lonch temps que han correguda fortuna ésser tornades a bon port» (tomo la transcripción de Escartí, La primera edició, ob. cit., p. 332). En la revisión del texto de la edición $d$ llevada a cabo en el taller de Claudi Bornat se corrigieron todos los castellanismos léxicos que se habían deslizado e incorporado al texto del impreso vallisoletano. Bornat tradujo al catalán todas las rúbricas de los poemas que Juan de Resa había puesto en castellano, aun cuando el texto de los poemas en la edición de Valladolid lógicamente estaban en la lengua original catalana. Ahora bien, Claudi Bornat lo que añade a su edición con respecto a los impresos de Barcelona, son los textos nuevos que incorporó Juan de Resa en su edición, que efectivamente no estaban en $b c$.

${ }^{32} \mathrm{El}$ cancionero $D$ fue el manuscrito que se tomó como base para la edición $b$. Aunque no fue sensu stricto un original de imprenta, ya que no fue un manuscrito confeccionado ex profeso como modelo para preparar la edición, sí fue utilizado y manipulado en el taller impresor con esa función; tal como revelan las marcas inconfundibles de su vida en el taller tipográfico de Carles Amorós. Véase al respecto los estudios ya citados de Lloret («La formazione di un canzoniere», art. cit.) y López Casas («iEl cancionero $D »$, art. cit.)

${ }^{33} \mathrm{He}$ realizado un estudio de la filiación de $d$ en «Las variantes de imprenta en la edición $d$ de Ausiàs March (Valladolid 1555)», en La poesía en la imprenta, ob. cit., pp. 79-94.

${ }^{34}$ Así lo dijo Ximeno en 1747 (Escritores del reyno de Valencia: Chronologicamente ordenados desde el año MCCXXXVII... hasta el MDCCXLVII, Valencia, Joseph Estevan Dolz, I, p. 41), y después Pagès, Les obres, ob. cit., I, p. 67: « $\mathrm{Si} \cdot 1$ treball den Carroz, ben manifestament destinat a la impressió, no va veure la llum, devia fer molt profit per l'edició de $1555 »$. Fuster también lo afirma comentando que quizás no llegó a la imprenta debido a la crisis de los talleres tipográficos en aquellos años: «El projecte de Lluís Carròs de Vilaragut, era, sens dubte, editar el recull de poemes de March [...] El manuscrit valencià del 1546, per algun camí desconegut per a nosaltres, anà a parar a Valladolid, i tot fa creure que va servir de base per a l'edició del 1555, el mateix any en què moria Carròs» (Fuster, «Lectures d'Ausiàs March», art. cit., p. 46).
} 
aunque no hay que descartar una copia intermedia, o que ambos copiaran independientemente de un antígrafo perdido). Pero sobre todo sabemos que Juan de Resa tuvo acceso al cancionero $E$ porque Jorge de Montemayor nos dice en su traducción que entre los originales que ha visto está el de Lluís Carròs de Vilaragut, que fue quien mejor entendió a Ausiàs $\operatorname{March}^{35}$, y dicho cancionero tuvo que llegarle a través de Juan de Resa y el círculo valenciano de Honorat Joan, obispo de Osma ${ }^{36}$. Sin embargo, de ahí a suponer que el impreso vallisoletano se basa en el cancionero de Carròs de Vilaragut hay un trecho. Solo el estudio textual ha podido arrojar luz sobre esta cuestión, y lo cierto es que el cancionero $E$ fue menos utilizado de lo esperado en la edición vallisoletana ${ }^{37}$. Con todo el asunto es complejo, porque cuando $d$ presenta una lección diferente a la de las ediciones $b c$, es decir, distinta de la tradición $D b c$ o $B D b c$, y coincide con $E$, resulta imposible saber si el impreso $d$ está copiando del manuscrito de Carròs o de algún otro testimonio que presenta la misma lección que él. En algunas ocasiones en que la edición $d-\mathrm{y}$ en la mayoría de los casos también $e$ - tienen la misma lección que el manuscrito $E$ frente a todos los demás testimonios, sí podemos decir que el editor de Valladolid podría haber copiado dicha lección del testimonio $E$ (o de otro testimonio perdido que leyera igual). Estas consideraciones de la filiación textual son necesarias para poder explicar más adelante qué sucede en los poemas $102 \mathrm{~b}$ y 103. Pero antes quiero referirme brevemente a la ecdótica humanística.

La difusión del Humanismo comportó una nueva técnica editorial, fundamentada en dos pilares: la colación de testimonios y la conjetura ${ }^{38}$. En la con-

${ }^{35}$ «Yo | me he llegado mas al que hizo tresla- | ladar el señor don Luis Carroz Bayle | general desta ciudad: por que segun to $\mid$ dos lo affirman, el lo entendio mejor que $\mid$ ninguno de los de nuestros tiempos», folio 3 .

${ }^{36}$ La vinculación entre Montemayor y Juan de Resa es conocida, de hecho el proyecto de traducir las obras de Ausiàs March se gestó en Valladolid, en la corte, donde el humanista valenciano Honorat Joan tenía un lugar destacado pues fue preceptor del príncipe don Carlos, hijo de Felipe II. Es muy probable que su relación con el obispo de Osma le abriera a Montemayor las puertas de Valencia y de la influyente familia valenciana Carròs de Vilaragut (emparentada con los Borja), pues fue allí donde publicó su traducción de March, así como la editio princeps de La Diana (parece que estuvo en Valencia entre 1558 o 1559-1560; véase López Casas, «Ausiàs March traducido», art. cit., pp. 305-306).

${ }^{37}$ Lo he estudiado en «El cançoner valencià de Lluís Carròs de Vilaragut i l'edició de les obres d'Ausiàs March a Valladolid», en Estudios sobre el Cancionero general (Valencia, 1511), ed. Marta Haro Cortés, Rafael Beltrán, José Luis Canet y Héctor H. Gassó, València, Universitat de València, 2012, 2 vols., II, pp. 653-668.

${ }^{38}$ Véase un estado de la cuestión en John F. D'Amico, Theory and practice in Renaissance textual criticism: Beatus Rhenanus between conjecture and history, Berkeley, University of California Press, 1988. Resumo directamente en esta explicación la primera parte del trabajo de Vicenç Beltran, «Ordenado y corregido por la mejor manera y diligencia. Hernando del Castillo, editor», en Filologia dei testi a stampa (Area iberica), ed. Patrizia Botta, Modena, Mucchi Editore, 2005, pp. 241-256, esp. pp. 241243. Beltran ilustra magistralmente toda la casuística de la conjetura aplicada a los textos romances en ejemplos concretos de versos de Jorge Manrique, de la Giuntina y del Laberinto de Fortuna. 
fección de libros latinos y vulgares en el Renacimiento se aplicaron ambos métodos, pero muy especialmente en las ediciones impresas; su carácter de empresa comercial, con amplia difusión en múltiples ejemplares, hacía que con frecuencia fueran sometidas a una esmerada atención, muy superior a la que acostumbraban a recibir las copias manuscritas. Los procedimientos de actuación eran, en primer lugar, la colación, usada para corregir los pasajes corruptos y para subsanar las lagunas del texto que había en los ejemplares, y en segundo lugar, en cuanto a la enmienda de errores concretos, frecuentes por la escasa competencia de los copistas, acostumbraban a utilizar la conjetura. La amplia aceptación de la conjetura, a pesar de las reservas teóricas y prácticas formuladas por los eruditos (más concretamente por Angelo Poliziano), fue uno de los elementos fundamentales de la crítica de aquel período en discusión filológica que podría alargarse hasta nuestros días.

Si nos fijamos en las ediciones hispánicas de poesía de la primera mitad del siglo Xvi, tenemos el valioso ejemplo del Cancionero General de Hernando del Castillo (Valencia, 1511, 1514). Vicenç Beltran ha realizado un estudio sobre el trabajo editorial de Castillo, que se inserta plenamente en la tradición ecdótica del Humanismo ${ }^{39}$. A partir del análisis de diferentes textos (de Los siete gozos de amor de Juan Rodríguez del Padrón, varios poemas de Juan de Mena y el romance Pésame de vos, el conde) afirma que la conjetura tuvo un papel primordial en el proceso de la constitutio textus de las obras del Cancionero General. Beltran, además de detectar enmiendas conjeturales, ha rastreado la contaminación, consulta y uso de fuentes secundarias tan frecuentes entre los humanistas cuando se trataba de subsanar lagunas ${ }^{40}$.

Las consideraciones precedentes nos permiten replantear la cuestión que anunciábamos: ¿existieron realmente los poemas $102 \mathrm{~b}$ y el 103 tal como nos los ha transmitido la edición de Valladolid? ¿Los compuso Ausiàs March con la forma que tienen en el impreso $d$ o son fruto de la re-creación del editor, o dicho de otro modo, de la creatividad de la imprenta, a partir del acceso a tradiciones textuales distintas, incluso quizá divergentes? ¿Se trata de poemas fantasma que jamás salieron de la pluma de Ausiàs March?

\footnotetext{
${ }^{39} \mathrm{Me}$ remito al estudio citado de Beltran en la nota anterior, al que hay que añadir, del mismo autor, «El Cancionero General (Valencia, 1511) y el Cancionero de la Biblioteca Británica», en Da Papa Borgia a Borja Papa. Letteratura, lingua e traduzione a Valencia, ed. Nancy De Benedetto e Ines Ravasini, Lecce, Pensa, 2010, pp. 121-150, donde desarrolla el sistema empleado por Castillo para seleccionar determinados textos del antígrafo compartido por LB1 y 11CG («LB1 y 11CG tomaron una parte de su contenido de un antígrafo común [...] LB1 y 11CG seleccionaron drásticamente el material que les resultaba más atractivo de este antígrafo, insertando (al menos 11CG) materiales de otras procedencias», pp. 146-147.

${ }^{40}$ «Como la mayor parte de los buenos editores de su tiempo [Hernando del Castillo] filtraba cuidadosamente sus fuentes, las sometía a una intensa crítica y las enmendaba por fin cuando sus lecciones no le resultaban satisfactorias» (Beltran, «Ordenado y corregido», art. cit., p. 250).
} 
Los manuscritos marquianos tardíos, desde el paso del XV al XVI o ya del XVI, como $F, B$ o $G$, presentan anotaciones que revelan la consulta de diferentes fuentes. El caso más complejo, y bien estudiado gracias a las investigaciones de Josep Lluís Martos, es el del cancionero $G$, cuyo análisis ha puesto de manifiesto no solo las diferentes secciones y manos que lo forman sino también la revisión e intervención de copistas sobre textos ya copiados en otras partes del cancionero ${ }^{41}$. El minucioso estudio de $G$ le ha permitido a Josep Lluís Martos adentrarse en los procesos y técnicas de recopilación de la poesía de March en el siglo XvI:

La mayoría de manuscritos parten de un antígrafo amplio, que bien se copia completo, o bien sirve para seleccionar materiales que faltan en la nueva recopilación. De esta manera, buena parte de los cancioneros de Ausiàs March tienen un núcleo que copia otro cancionero o, incluso, se trata físicamente de una recopilación anterior, que se reutiliza y se completa con la adición de nuevos cuadernos provenientes de las copias de otros materiales. ${ }^{42}$

El cancionero $E$ sería el caso más extremo en este sentido, puesto que llega a entremezclar tradiciones incluso en un mismo poema, cosa que como veremos también sucede en la edición $d^{43}$.

Veamos qué sucede en el poema $102 \mathrm{~b}$ de la edición vallisoletana. El poema, que Archer edita como independiente - tal como figura en $d$ - asignándole una numeración propia que no estaba en la edición de Pagès, parece una «esparsa» creada a partir de la segunda estrofa del 102:

És fàcil que el compilador de l'edició $d$ hagi creat una «esparsa» a base de la versió dels vs. 9-16 que trobem a $B D b c$, canviant tan sols, al v. 1 «aquest»

${ }^{41}$ Josep Lluís Martos, «Cuadernos y génesis del Cancionero $O^{l}$ de Ausiàs March (Biblioteca Universitaria de Valencia Ms. 210)», en Actas del II Congreso Internacional «Cancionero de Baena», ed. Jesús L. Serrano Reyes, Baena, Ayuntamiento de Baena, 2003, 2 vols., II, pp. 129-142; íd., «La duplicació de poemes en el cançoner G d'Ausiàs March», Cancionero General, 7 (2009), pp. 35-69; íd., «La gènesi del Cançoner $G$ de Ausiàs March: les mans dels copistes de G2 y G4», en Actas del XIII Congreso Internacional de la AHLM, ob. cit., 2010, 2 vols., II, pp. 1349-1359.

${ }^{42}$ Martos, «La copia completa», art. cit., p. 15. También son imprescindibles en este sentido las investigaciones de Beltran sobre la formación del Cancionero de Ausiàs March, en Poesia, escriptura $i$ societat, ob. cit., y en «Tipologia i gènesi dels cançoners. La reordenació de $J$ i $K$ », Llengua \& Literatura, 11 (2000), pp. 355-395.

${ }^{43}$ Es lo que Pagès llamó «eclecticismo», en cuanto a que entremezclan tradiciones: «Però alguns, sobre tot els del $\mathrm{XVI}^{\text {en }}$ segle, són obres eclectiques per a les quals han sigut posats a contribució $\cdot 1 \mathrm{~s}$ manuscrits més antics y adhuc les primeres edicions [...] Les cinc edicions del $\mathrm{XvI}^{\mathrm{en}}$ segle estan en el mateix cas. Adhuc quan es possible lligar-les a un manuscrit o a una edició constituint, per dir-ho així, llur prototip, ofereixen variants que no provenen pas solament dels editors mateixos, sinó també dels manuscrits y dels impresos anteriors», Pagès, Les obres, ob. cit., I, p. 118. 
per «molt trist», i al v. 2 «passionat» per «apassionat» (la forma «passionat» reapareix a l'edició $e$ ). No obstant això, no podem descomptar que la cobla hagi circulat en vida del poeta com a esparsa ${ }^{44}$.

De todas formas, parece más bien que a Juan de Resa le llegaron las dos tradiciones textuales en lo que concierne a estos versos, y no quiso renunciar a ninguna de ellas, creando un «nuevo» poema: una «esparsa» que incluyó en la sección de «esparsas de amor» en su reordenación del cancionero, cuando creó una sección especial para ellas. Esta explicación, a mi entender, plausible, se apoya en la filiación textual y en el valor que le da Juan de Resa a los diferentes testimonios que vio y consultó. Por un lado, tiene la tradición de $D B b c$ que es la que sigue como base para el conjunto de la edición y también para el poema 102 al que nos estamos refiriendo, y por otro, como tiene acceso a otras fuentes, observa una estrofa 2 en el poema 102 totalmente distinta (la que comparten $F G_{1} E H K a$ ). En este caso la fuente podría haber sido el propio cancionero $E$-que por lo que parece pudo haber consultado- o cualquier otro de los de la otra tradición que leen como $E$ en estos versos 9-16. Ante la disyuntiva de editar una u otra versión, decide desgajar la estrofa 2 de la tradición $D B b c$, y cambiando parte solo del primer hemistiquio del primer verso Yo so aquest por Yo so molt trist crear un poema nuevo. La cuestión que quiero remarcar es que en la tradición $F G_{1} E H K a$ está el impreso de Baltasar de Romaní, la editio princeps, que entre los 46 poemas que la componen incluye el 102. A partir del estudio que he realizado de la tradición textual de $d$, si las variantes que encuentra el editor de $d$ están también en $a$ casi sistemáticamente opta por dicha lección. No hay que olvidar que es posible que el prestigio del Comendador Escrivá como poeta fuera uno de los elementos que pesara en la decisión de Juan de Resa, y si además es compartida con $E$, mejor aún ${ }^{45}$. Por tanto, yo pienso que el poema $102 \mathrm{~b}$ fue creado en el proceso de revisión de fuentes que se hizo al preparar la edición $d$, y no debe de extrañarnos que dentro de un mismo poema se opte por una tradición distinta

\footnotetext{
${ }^{44}$ Ausiàs March, Obra completa, ed. cit., 2, p. 414. Parto siempre de la edición de Robert Archer (2. Apèndix), que nos ha facilitado en gran manera el estudio de las variantes. Sin embargo, en el caso de $E$ y $D$, o para determinados lecciones o lugares críticos reviso siempre los testimonios directamente o a través de copias digitalizadas. Ya se pueden consultar prácticamente todos los testimonios en el servidor de la Biblioteca Virtual Miguel de Cervantes (<http://www.lluisvives.com/bib_autor/ausiasmarch/>). Las ediciones, también en la Biblioteca Valenciana Digital.

${ }^{45}$ El prestigio de Baltasar de Romaní, el Comendador Escrivá está presente tanto en $d$ como en el manuscrito $E$, que fue corregido en varios pasajes con las lecciones de $a$. La identificación entre Baltasar de Romaní y el Comendador Escrivà en Ivan Parisi, «La verdadera identidad del Comendador Escrivà, poeta valenciano de la primera mitad del siglo XVI» Estudis Romànics, 31 (2009), pp. 141-162.
} 
como sucede a menudo en $E$, en $d$, en $G$ e incluso en $D$, como ha demostrado Josep Lluís Martos en su estudio del poema $87^{46}$.

Estrofa 2 poema 102 testimonios FG $\mathrm{EHKad}$

Traure nos [Fugir no Ede] pot dauer nom de mesqui

Qui son uoler la ental part [prat a] lancat

Que no sab com ama o es amat

Doch o deno no pot fer [far $d]$ bon juhi

E passa molt dolrossa [fort dollor e $H K a$ ] passio

Quen pert dormir e sen ueu alterat

En vn instant se troba reposat [y en vn $E]$

Quel par james vendra en tal saho

\section{Estrofa 2 poema 102 testimonios DBbc}

Yo so aquest /e puch me dir mesqui

Doy, e damor /me trob passionat [pationat $B$ ]

$\tan$ egualmen /e fort que so

[questich $b c$ ] torbat

qual daquests / dos ha maior part en $\mathrm{mi}$

Dant me assalt / qual seuol passio ab tal dolor / quen estich alterat E puix [puys $B$ ] me trob / daquella reposat Venint apres / del altra la [lur $b c]$ saho
Poema $102 b$ testimonios de

Yo so molt trist / e puch me dir mesqui

De oy ed'Amor/me trob apassionat [passionat $e$ ]

tant egualment / e fort que'stich torbat

qual de aquests dos / an maior part en $\mathrm{mi}$

Dant me asalt / qualseuol passio $\mathrm{ab}$ tal dolor / quen estich alterat

e puix me trob / de aquella reposat

vinint a pres / del altra llur saho

En cuanto al poema 103, la cuestión es algo más compleja, pero nos remite de nuevo a una doble tradición textual. El poema 103 se ha conservado en tres versiones distintas. La primera, que consta de 60 versos -7 octavas + una «tornada» de 4 versos- está en los testimonios FBDEHKbc. La segunda, con 84 versos, solamente figura en el cancionero $B$ (que tiene asimismo la primera versión como hemos dicho). Esta segunda versión incorpora 3 estrofas nuevas entre la $6^{\mathrm{a}}$ y la $7^{\mathrm{a}}$. Los versos añadidos están intercalados en dicha posición seguramente porque la estrofa 7 no podía cambiar de lugar, ya que es en dicha estrofa donde queda de manifiesto que el poema es una «resposta» a una demanda perdida. Ausiàs March parece responder a una demanda en la que su autor solicita su consejo sobre cómo debería emplear su riqueza un amigo suyo ${ }^{47}$. Y la tercera versión, también de 84 versos, transmitida por las ediciones $d e$, que en cierta forma fusiona la primera y la segunda versión. Y esto es lo que analizaremos a continuación para entender cómo se ha creado o re-creado este último poema, seguramente fruto de la intervención de Juan de Resa, el editor de $d$.

El impreso $d$ sigue en general su tradición textual ${ }^{48}$, la que llega de $D b c$, con quien se agrupa también $B$ (en su primera versión) y a veces $E$, aunque

\footnotetext{
${ }^{46}$ Josep Lluís Martos, «Tot entenent amador mi entengua: la transmissió del poema 87 en el cançoner $G$ de Ausiàs March», Catalan Review, 24 (2010), pp. 59-77.

${ }^{47}$ Primer verso de la estrofa 7: «Per consegüent, lo meu consell jo don:», Ausiàs March, Obra completa, ed. cit., 2, p. 459.

${ }^{48} \mathrm{Me}$ refiero solo a $d$, porque el editor de $e$, Claudi Bornat, en este poema se limita a seguir fielmente
} 
este cancionero $E$ a partir de la estrofa 4 forma grupo con $F H K$ hasta el final del poema. Recojo solamente las variantes de mayor entidad ${ }^{49}$ :

11 per aquells $F H K$ : quels pusca $B D E b c d e$

12 no sabent $F H K$ : ignorant $B D E b e$ : ignarant $c$ : ignyorant $d$

21 sens gran cost $F H K$ : e sens cost $B D E b c d e$

22 nostrens ho $F H K$ : les coses $B D E b c d e$

25 per [que $K$ ] null temps $F E H K$ : compost no fartarem $B D b c d e$

26 que passa dom lo delit natural $F E H K$ : quant passa dom lo terme natural BDbcde

$27 \mathrm{E}$ sols aquest es lo mer $F H K$ : E sols aquest es lo meu $E$ : De la raho nax e del BDbcde

$28 \mathrm{Y}$ aquell maior que dentendre prenem $F E H K$ : Aquests reglats en termens lo meterem $B:$ Aquests reglats en termens lo [los $e]$ metrem $\mathrm{Dbcde}$

33 es molt menys que [quel $K]: F E H K d e:$ pot esser dit $B D b c$

34 No resemblant [no resemblat $K$ ] : FEHKde: Com plau e mou $B$ : Complau e nou $\mathrm{Dbc}$

35 semblals [semblas $K$ ] mas poch $F E H K d e$ : Dells lesser pren $B D b c$

37 ociu FEHK : destruu BDbcde

39 Matant lo cors FEHKde : Perço los pechs $B D b c$

40 E quill segueix a ell yassi desplau FEHKde: Han per dolent qui daquell se retrau $B:$ han per dolent qui de aquell [daquell $D$ ] se trau $D b c$

42 E menys de grat aquest se fa servir $F E H K$ : Donal dolor pels desitxs no complir BDbcde

43 Car per aquest lome no pot venir $F E H K$ : Senyor lo fa subiugat a servir $B D b c d e$ $44 \mathrm{Al}$ desijat e fa lo queli plau $F E H K$ : E pel costum no sentiu que passau $B D b c d e$ 48 Les $[$ Los $K]$ fins d'aquest $F H K$ : sa fi no es $B D E b c d e$

Fijémonos que solamente en la estrofa 5 (versos 33-40), de comparten lecciones con el grupo $F H E K$, frente a $B D b c$, pero a lo largo del poema, son la excepción.

La segunda versión del poema 103, la de 84 versos que solamente figura en $B$, está situada al final del códice, en el verso del folio 172, cuando ya el copista había dado por finalizada la copia, pues consta deo gratias en el recto del $172^{50}$. Lo cual significa que se incorporó al códice en otro momento, ya

el texto del impreso $d$.

${ }^{49}$ Pongo en negrita el número de verso y agrupo los versos por estrofas.

${ }^{50}$ En $B$ la primera versión del poema 103 (60 versos) ocupa los folios $102 \mathrm{v}-103 \mathrm{v}$. El Cancionero $B$ fue copiado por Pere Vilasaló en 1541 para Ferran Folch de Cardona, tal como figura en el colofón. 
a partir de otros materiales, distintos del antígrafo que había sido copiado. Por alguna razón, quizás por ser más largo o por ser bastante diferente en sus versos, se tomó la decisión de copiarlo de nuevo. Efectivamente esta segunda versión, dejando al margen las 3 estrofas nuevas, presenta un texto distinto en muchos casos al de la primera versión. Pongo en primer lugar el verso según la segunda versión de $B$ y a continuación la lección de $B$ y de los que leen con él, para poner de manifiesto cuán distintas son muchas lecturas:

21 Dins nos sta lo be que obs hauem $B^{2^{a}}$ : prest e sens cost es quant mester hauem $B D E b c d e$ : Prest sens gran cost es quant mester hauem $F H K$

23 La voluntat ab conçell elegex $B^{2^{2}}$ : Tant quela fam com mes nauem nos [hauem nos $D b c d e]$ creix : $D F H K b c d e$ : Tant que la fam com mes hauem mes crex $B E$

$24 \mathrm{O}$ sens concell pren lo mitx o lestrem $B^{2^{a}}$ : Dalguns desigs complits fastig nauem $F$ : Dalguns desigs complits fastigs reebem $H K$ : Dalgun desitx complit fastitx reebem $B D E b c d e$

25 conciliatiu direm $B^{2^{2}}$ : compost no fartarem $B D b c d e$ : per [que $K$ ] null temps FEHK

26 Lenteniment sera dit volitiu $B^{2^{a}}$ : quant passa dom lo terme natural $B D b c d e$ : que passa dom lo delit natural FEHK

27 Del apetit qui es dit $B^{2^{a}}$ : De la raho nax e del $B D b c d e$ : E sols aquest es lo mer $F H K$ : E sols aquest es lo meu $E$

$28 \mathrm{Amb}$ ells concort virtut moral traurem $B^{2^{2}}$ : Aquests reglats en termens lo meterem $B$ : Aquests reglats en termens lo [los $e]$ metrem $D b c d e: Y$ aquell maior que dentendre prenem $F E H K$

29 Cell no es res si a ells no ha $B^{2^{a}}$ : Quant [Quants $F$ ] aquests han lo seu propi BDFEHKbcde

30 Ells valen poch si nol tenen restret $B^{2^{4}}$ : Lome pot dir ques en lo mon content BDFEHKbcde

31 A dretant sa no fartabla set $B^{2^{a}}$ : La donchs lo uer coneix lenteniment BDFEHKbcde

33 Tal apetit $B^{2^{2}}$ : Aquest voler $B D b c d e$ : Aquell voler $F E H K$

34 Es produyt marauellosament $B^{2^{n}}$ : Com plau e mou [nou $D b c$ ] als quil an engendrat $B D b c$ : No resemblant [resemblat $K$ ] als qui lan engenrat $F E H K d e$

35 Lo finit fa ab linfinit vn ent $B^{2^{a}}$ : Dells lesser pren de que lom es torbat $B D b c$ : Semblals [Semblas $K$ ] mas poch de quel om es torbat FEHKde

36 E sa virtut cascu ab laltra part $B^{2^{a}}$ : vehent dell fets lançant [lancants $\mathrm{FH}$ ] natura part $B D F E H K b c d e$

Sobre $B$ véase Pagès, Les obres, ob. cit., pp. 14-17, y Beltran, Poesia, ob. cit., pp. 153-155, así como Philobiblon: <http://sunsite.berkeley.edu/Philobiblon/phhmbi.html>, manid 1967. Sobre la historia y formación del cancionero, véase además Josep Lluís Martos, «De la filología material a los textos y sus variantes: el proceso de copia del cancionero $B$ de Ausiàs March» (en prensa); y para el estudio del códice, íd. «La estructura codicológica del cancionero $B$ de Ausiàs March» (en prensa). 
38 Quant es amich $B^{2^{a}}:$ E les fill cert $B D F E H K b c d e$

40 Quel rational $B^{2 a}$ : Han per dolent $B D b c$ : E quill segueix FEHKde

41 ñe per aquest $B^{2^{a}}$ : aquest lo fa BDFEHKbcde

42-43 $B^{24}$ invierte el orden de los versos

44 James a port atteny la sua nau $B^{2^{2}}$ : E pel costum no sentiu que passau $B D b c d e$ : Al desijat e fa lo queli plau FEHK

45 Ell portab si vn altre quil confon $B^{2^{a}}$ : Ell [Çell $\left.E d\right]$ es aquell qui lom portan lo mon BDFEHKbcde

47 per null temps y vendra $B^{2^{a}}:$ james hi peruendra $B D F E H K b c d e$

49/73 ${ }^{51}$ dare $B^{2^{a}} d e$ : yo don $B D F E H K b c$

$\mathbf{5 2 / 7 6}$ ason stat li ve $B^{2^{a}}$ : al necessari son $B D F E H K b c$ : al neçessarils te de

$\mathbf{5 4 / 7 8}$ lobesta en dar enon tenir $B^{2^{2}}$ : non traura daquells son propi be $B D F E H K b c d e$

55/79 Aço no pot home auar sentir $B^{2^{4}}$ : Hoc hun be fals que tost sen ua o ue BDFEHKbcde

56/80 Entendre si e creurab dolor gran $B^{2^{a}}$ : Tal quel [qual $K$ ] pus rich es lo mes pobreiant $B D F E H K b c d e$

58/82 Contentament $B^{2^{4}}:$ Lo gran delit $B D F E H K b c d e$

$\mathbf{5 9 / 8 3}$ sperança $B^{2^{2}}$ : cobeiança $B D F E H K b c d e$

Lo primero que se observa es que en un único caso la edición $d$ comparte lección con $B^{2^{4}}$, y se trata de la palabra rima de la última estrofa antes de la «tornada». El editor del impreso $d$ se ve inducido a realizar dicho cambio, porque al intercalar las 3 estrofas nuevas como el testimonio $B^{2^{a}}$ se ve obligado a hacer rimar el primer verso de la estrofa $7 / 10$ con el último verso de las estrofas añadidas ( 73 dare $B^{2^{2}} d e: 49$ yo don $\left.B D F E H K b c\right) .{ }^{52}$ Este cambio en la palabra rima provoca también la sustitución de la última palabra del cuarto verso de la estrofa $7 / 10$ que debe rimar con dare, en este caso el editor no recurre a $B^{2^{a}}$, sino que pone otra lección ( 76 ason stat li ve $B^{2^{2}}: 76$ al neçessarils te $d e: 52$ al necessari son $B D F E H K b c$ ).

Lo que queda claro es que el impreso $d$, pese a tener 84 versos como la segunda versión de $B$, no comparte lecciones con $B^{2^{a}}$ en esta parte del poema. Veamos qué sucede en las 3 estrofas añadidas en $B^{2^{a}} \mathrm{y}$ de. Paso a presentar las variantes de cierta entidad:

56 encontrar $B^{2^{a}}:$ contrastar de

\footnotetext{
${ }^{51}$ Doble numeración de los versos porque se trata de la última estrofa y «tornada». El primer número se refiere a la posición del verso en $F B D E H K$, y el segundo, a la posición que tiene en $B^{2^{a}} d e$.

${ }^{52}$ Último verso de la estrofa 9 de $B^{2^{a}} d e$, el 72: «No per scrits mas parlant ho dire».
} 
$59 \operatorname{lom} B^{2^{a}}$ : lo mon de

65 via $B^{2^{\mathrm{a}}}$ : guia de

En 24 versos solamente hay estos tres cambios de tipo léxico. Todos podrían haberse producido en el proceso de memorización y copia. Teniendo en cuenta que la segunda versión de $B$ (la que he convenido en denominar $B^{2^{a}}$ ) tiene en el resto del poema muchas lecciones singulares como hemos visto, incluso 3 y 4 versos enteros, llama la atención que estas tres estrofas añadidas en $B^{2^{2}}$ y en de sean, desde el punto de vista textual, tan semejantes. Todo ello me inclina a pensar que el editor del impreso vallisoletano tuvo a su alcance o el propio cancionero $B$ o una copia muy cercana a él con la segunda versión del poema 103 (84 vv.), y que tomó de él exclusivamente las 3 estrofas que no estaban en su tradición textual, la de $B D b c$, pues para el resto del poema no sigue a $B^{2^{a}}$, excepto para el verso en que se ve obligado a cambiar la rima a consecuencia de añadir las estofas nuevas.

Lo sucedido con estos poemas 102 b y 103 en la edición vallisoletana de Juan de Resa ilustra los mecanismos de creación, reunión y fusión de materiales que tuvieron lugar en las recopilaciones tardías de la poesía de Ausiàs March, mecanismos que habrá que ir estudiando en casos concretos, como los que hemos analizado aquí. En la misma línea estaría la fusión de los poemas 29 y 30 , producida en el proceso de copia, seguramente debido a la ausencia de rúbricas en los cancioneros más antiguos. De nuevo es Juan de Resa, quien en su lectura y revisión atenta, detectó el error en la rima y optó por modificar la palabra rima que no concordaba. Todos estos procesos, analizados individual y conjuntamente en relación a todos los testimonios conservados, nos darán la medida para poder editar mejor la poesía de Ausiàs March, pues nos acercan a un mejor conocimiento de lo que verdaderamente escribió el poeta valenciano. Además nos ilustran en los fenómenos a los que hemos de atender cuando estudiamos, analizamos o editamos los textos medievales que tuvieron vida, gracias a la imprenta, en los siglos XVI y XVII ${ }^{53}$.

Recibido: 6/07/2014 Aceptado: 21/09/2014

\footnotetext{
${ }^{53}$ Sobre la problemática de la intervención en los textos impresos, veáse el texto de Josep Lluís Martos «Hacia un canon de transmisión de la poesía antigua en la imprenta», en La poesía en la imprenta antigua, ob. cit., pp. 11-12. El libro es fruto de las investigaciones del proyecto La variante en la imprenta: hacia un canon de transmisión del cancionero y del romancero medievales, (FFI2011-25266, Ministerio de Economía y Competitividad), el cual es, en cierto sentido, continuador del proyecto de investigación Del impreso al manuscrito: hacia un canon de transmisión del cancionero medieval (FFI2018-04486, Ministerio de Ciencia e Innovación). De ambos es investigador principal y alma máter Josep Lluís Martos.
} 
$\cos$

DE LA DOBLE VERSIÓN AL POEMA FANTASMA: LA CREATIVIDAD DE LA IMPRENTA

RESUMEN: Tras una introducción sobre los cambios que introdujo la imprenta en la difusión de la literatura medieval y en su textualidad, se analizan dos poemas de Ausiàs March (el 102b y el 103) en la forma que han sido transmitidos por la edición de Juan de Resa (Valladolid, 1555). Plantea la posibilidad de que dichos poemas sean producto de una operación editorial a partir del conocimiento por parte de Juan de Resa de dos tradiciones textuales totalmente diferentes, sin querer renunciar a ninguna de ellas.

Palabras ClavE: Ausiàs March. Transmisión textual. Imprenta. Impresos.

FROM THE DOUBLE VERSION TO THE GHOST POEM: CREATIVITY OF PRINTING

ABSTRACT: After an introduction on the changes introduced by the printing press in the diffusion of medieval literature and its textuality, two Ausiàs March's poems (102b and 103) are analysed as they have been transmitted by the edition of Juan de Resa (Valladolid, 1555). The paper suggests the possibility that these poems are a product of a publishing operation based on Juan de Resa's knowledge of two completely different textual traditions, not wanting to give up any of them.

KEYwords: Ausiàs March. Textual transmission. Printing. Printed books. 